\title{
QUANTUM-CLASSICAL CORRESPONDENCE IN INTENSE LASER FIELD-ATOM INTERACTIONS
}

\author{
C.M. Bowden, S.D. Pethel \\ Weapons Sciences Directorate, AMSMI-RD-WS-ST \\ Research, Development, and Engineering Center \\ U.S. Army Missile Command \\ Redstone Arsenal, AL 35898-5248, USA
}

A.T. ROSENBERGER

Department of Physics and Center for Laser and Photonics Research

Oklahoma State University, Stillwater, OK 74078-3072, USA

AND C.C. SUNG

Department of Physics, University of Alabama in Huntsville

Huntsville, AL 35899, USA

High-field ionization suppression in a classical Kepler ensemble is discussed in terms of optimization with respect to pulse turn-on rate as well as pre-pulse preparation. It is argued that high-field ionization suppression is best understood in terms of reduced probability of ionization for pulsed fields, whereas for a quasi-steady field, high-field ionization suppression implies a reduced ionization rate at higher intensities. The classical ensemble is used to calculate the high-field ionization rate of a one-dimensional atomic model using a Gaussian short-range potential and the results are compared with high-frequency Floquet theory results recently reported by other authors. Better than qualitative agreement is found and the results are compared and discussed in terms of quantum superposition and classical interference. Finally, high-field ionization suppression is discussed in relation to statistical relative stability of classical orbits of the ensemble, and classical interference for both short- and long-range potentials. Correspondence with quantum superposition is interpreted in relation to quantum-classical correspondence.

PACS numbers: $32.80 . \mathrm{Rm}, 42.50 . \mathrm{Hz}$ 


\section{Introduction}

The possibility of atomic stabilization in an intense optical field has been an area of active theoretical research in recent years $[1,2]$. Both quantum and classical approaches have been taken, using many different model atomic potentials. We and our collaborators have studied several classical-ensemble models for intense-field stabilization and the correspondence between their results and those of quantum treatments [3-10]. This report is a synopsis of our most recent results [7-10].

Seemingly contradictory results can be found in the literature [2], and there is not universal agreement that the phenomenon of atomic stabilization in an intense laser field even exists. Also, those who do feel that the evidence supports its existence are not always in agreement on the conditions for its manifestation. We have attempted to clarify this situation in our recent work. Part of the disagreement stems from a potential ambiguity in the meaning of "stabilization". Some authors interpret it strictly, so that it refers to an ionization probability that is less than unity or that decreases with increasing field; in this strict interpretation, it is inappropriate to describe a decreasing ionization rate as stabilization, unless the rate decreases to zero. Many other authors, however, would prefer to state that any reduction in ionization rate makes the atom more stable. To avoid this possible ambiguity, we will use the term "high-field ionization suppression" (HFIS); this term can refer to either a reduced ionization rate or a probability of ionization that is less than unity. The interpretation of HFIS as a reduced rate of ionization makes sense if the field is in a quasi-steady state (changing no faster than adiabatically); this is because the ionization rate $\Gamma\left(\alpha_{0}\right)$ is determined by the field strength $\left(\alpha_{0}=A / \omega^{2}\right.$, where $A$ is the field amplitude and $\omega$ is its frequency). Only when the field has a finite duration, as in the case of a pulse $\left(\alpha_{0}(t)\right)$, is the interpretation as a reduced ionization probability meaningful, and then the probability of ionization is given by

$$
P=1-\exp \left\{-\int \Gamma\left[\alpha_{0}(t)\right] \mathrm{d} t\right\},
$$

where the ionization rate $\Gamma$ is integrated over the pulse duration.

Most of the apparent contradictions in the literature can be resolved by taking care in making comparisons, as there are many parameters whose variation can lead to qualitatively different results. For example, in considering ionization rates, one must ask how the atom is excited to a quasi-steady state $[6,7,11,12]$. In the case of pulsed fields, the probability of ionization depends very much on the pulse shape, particularly on how the pulse turns on (ramp-up); ionization is most likely to happen during ramp-up $[7,8]$. One must also consider the range of the potential $[6,9,10]$ and its dimensionality. The angular momentum of the initial state is important, as is the field frequency relative to the binding energy. One must also be careful in using a criterion to define when ionization occurs. In spite of the great diversity of results reported in the literature, quantum-classical correspondence [10] indicates that the various theoretical methods predicting HFIS [2] may actually have more in common than previously thought.

In the next section, we present a treatment of HFIS as a classical Kepler problem and emphasize how HFIS can be enhanced by proper tailoring of the field 
pulse. The reduction of ionization rate in a short-range potential is presented in Sec. 3. Section 4 contains a discussion of the classical interpretation of HFIS in terms of statistical relative stability of orbits, and we conclude with a discussion of our results and quantum-classical correspondence in Sec. 5 .

\section{Classical Kepler ensemble}

We use the Kepler model to write the classical equations of motion for atomic hydrogen in cylindrical coordinates $(\rho, z)$ and atomic units $\left(e=m_{\mathrm{e}}=\hbar=1\right)$ :

$$
\begin{aligned}
& \frac{\mathrm{d}^{2} \rho}{\mathrm{d} t^{2}}=-\frac{\partial V}{\partial \rho}+\frac{m^{2}}{\rho^{3}}, \\
& \frac{\mathrm{d}^{2} z}{\mathrm{~d} t^{2}}=-\frac{\partial V}{\partial z}+\eta(t) A \sin (\omega t),
\end{aligned}
$$

where $V(\rho, z)=-1 / r=-1 /\left(\rho^{2}+z^{2}\right)^{1 / 2}$ is the potential function and $m$ is the azimuthal angular momentum. The external field $\varepsilon(t)=\eta(t) A \sin (\omega t)$ is specified by the maximum amplitude $A$ and frequency $\omega$, and it takes the form of a trapezoidal pulse whose envelope is given by

$$
\eta(t)= \begin{cases}t / T_{0}, & 0 \leq t \leq T_{0}, \\ 1, & T_{0} \leq t \leq T, \\ \left(T+T_{0}-t\right) / T_{0}, & T \leq t \leq T+T_{0} .\end{cases}
$$

The ramp slope, $R$, defined by

$$
R \equiv \frac{A}{T_{0}}
$$

is an important parameter. We solve Eqs. (2) and (3), using $\omega=1$ and $m=0.75$. The roles played by $\omega$ and $m$ were discussed earlier [6,7]. We fix the length of the pulse plateau, $T-T_{0}$, at 30 optical periods. The initial conditions determine whether or not a particle will be ionized; we take a distribution of initial conditions

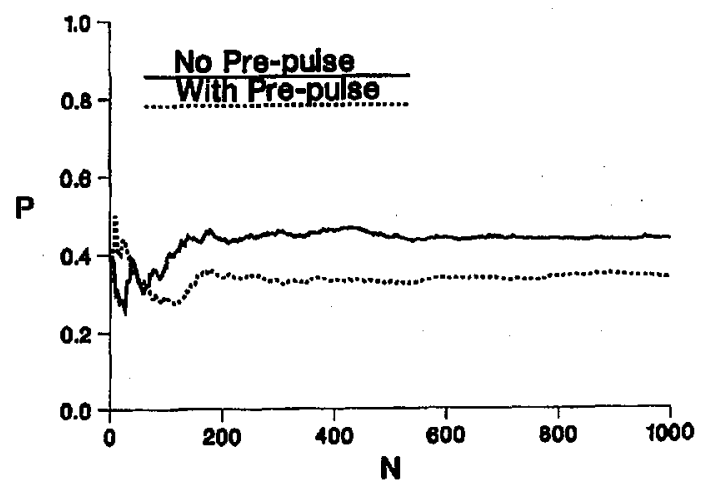

Fig. 1. Ionization probability $P$ versus the number of particles in the ensemble $N$. A pre-pulse (described in the text) is seen to reduce $P$ from 0.44 to 0.34 . 
consistent with $r=\left(z^{2}+\rho^{2}\right)^{1 / 2}=1$, where $\rho \leq 0.75$, and a total (ground state) energy of $E=-0.5$. A particle is considered to have ionized if it has a positive total energy at the end of the pulse.

A discussion of the process of ionization will be given in Sec. 4. Here we present some results showing how to enhance HFIS by tailoring the shape of the pulse. We use an ensemble of 200 members; Fig. 1 shows this to be sufficient. In Fig. 1, the probability of ionization (fraction of the ensemble that ionizes) is shown as a function of ensemble size for the case of $A=8, R=2$. Also shown in this figure is the enhancement of HFIS that results from preceding the main pulse with a small trapezoidal prepulse with $A=0.5, T_{0}=0.25$ periods, and $T-T_{0}=1.5$ periods - the effect of the prepulse is to move the electron to a larger orbit, making it less likely for the electron to pass near the nucleus during the critical ramp-up phase of the field. We have found that most ionizations take

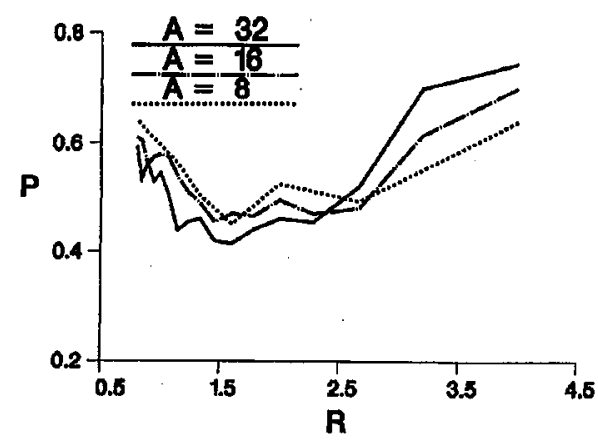

Fig. 2. Probability of ionization during ramp-up, as a function of ramp rate, for $m=0.75$.

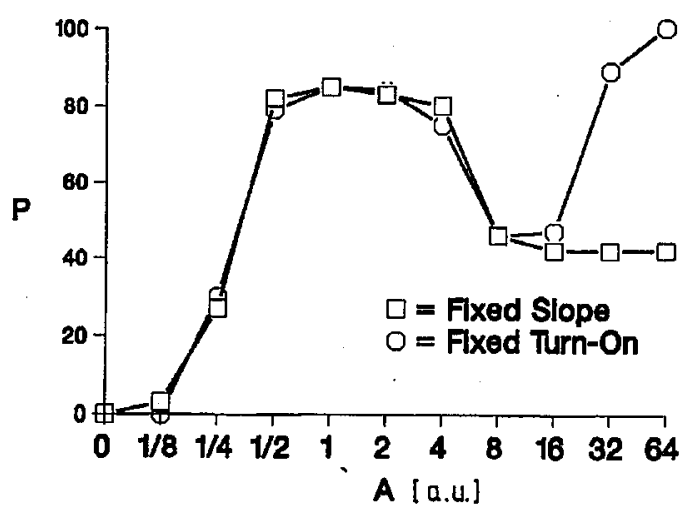

Fig. 3. Percent ionization $P$ versus field amplitude $A$. Open squares represent a fixed turn-on slope $R=2$. Open circles represent a fixed turn-on time of 4 periods. Ionization returns to $100 \%$ in the latter case because the slope $R$ becomes very steep as $A$ increases. 
place during ramp-up, and that the ramp rate $R$ is critical: too slow, and the electron spends too much time in the "death valley" region, of highest ionization rate, around $A=2$ [13]; too fast, and the electron can be subject to a high field while still close to the nucleus. We have shown $R=2$ to be the optimal value, producing the least ionization [7]. This is illustrated in Figs. 2 and 3. Shown in Fig. 2 is the probability of ionization during ramp-up vs. ramp-up rate for three values of $A$ larger than the "death valley" value of $A=2$, clearly demonstrating an optimum turn-on rate of $R=2$. In Fig. 3, the percent ionization (for a pulse with no pre-pulse) is given for different values of $A$, both for constant $T_{0}=4$ (periods) and for constant $R=2$. Some apparently contradictory results in the literature are probably a result of these two different choices.

\section{Classical HFIS in a short-range potential}

A recent paper [14] calculates high-constant-field ionization rates using the approximate solution to the Schrödinger equation given by high-frequency Floquet theory (HFFT). The (short-range) potential used is a Gaussian well with one bound state, as treated in previous quantum calculations [15, 16]. An interesting feature of the results, for sufficiently large fields, is that the ionization rate decreases non-monotonically with increasing field strength. (This behavior has also been found in a long-range potential, the one-dimensional soft Coulomb potential [17].) The same results can be obtained by a classical calculation [10], and the agreement with quantum methods is better than merely qualitative.

The results of Ref. [14] are found by an adiabatic quantum method and represent HFIS in a short-range potential. Field-atom interaction in the HFFT may be thought of as a perturbation to the steady-state Kramers-Henneberger $(\mathrm{K}-\mathrm{H})$ potential $[1,2]$, which is the time average, in the infinite-frequency limit, of the potential $V(u)$ given below. The ionization rate of interest is that of the $\mathrm{K}-\mathrm{H}$ ground state, which has been reached from the Gaussian well's bound state by adiabatic turn-on of the field. We wish to approximate this method classically, and to do so we solve the classical equation of motion (in atomic units) for an electron in a 1D potential $V$ and subject to a field of amplitude $A$ and frequency $\omega$,

$$
\ddot{x}=-\frac{\partial V}{\partial x}-A \cos (\omega t),
$$

by making the transformation

$$
u=x-\frac{A}{\omega^{2}} \cos (\omega t)
$$

so that the equation of motion becomes

$$
\ddot{u}=-\frac{\partial V}{\partial u} .
$$

In Eq. (8), the potential to be considered here is

$$
V(u)=-0.27 \exp \left\{-\left[u+\alpha_{0} \cos (\omega t)\right]^{2} / 4\right\},
$$

where $\alpha_{0}=A / \omega^{2}$. We take $\omega=0.236$ a.u., as in Refs. [14-16]. We begin with a large number of particles with a random, uniform distribution of initial conditions $(u(0), \dot{u}(0))$, such that the initial energy of each is $E(0)=-0.13$, equal to that 
of the bound state of $V$ for $\alpha_{0}=0$ [14]. This ensemble is meant to approximate, in an average sense, the corresponding quantum state. The field is then ramped linearly from zero to its final value at a constant rate, chosen to be slow enough (e.g., $\mathrm{d} \alpha_{0} / \mathrm{d} t=0.5 /$ optical period $=0.019$ a.u.) that this process approximates adiabatic evolution to the perturbed ground $\mathrm{K}-\mathrm{H}$ eigenstate. The members of the initial ensemble that survive the ramp-up of the field (remain unionized) then constitute an ensemble approximating the perturbed $\mathrm{K}-\mathrm{H}$ ground state. Finally, while the field amplitude remains constant (hence constant $\alpha_{0}$ ), the decay of the number of survivors is analyzed to find the ionization rate $\Gamma\left(\alpha_{0}\right)$; a particle is considered to be ionized when the absolute value of its coordinate $u$ becomes greater than $4 \alpha_{0}$.

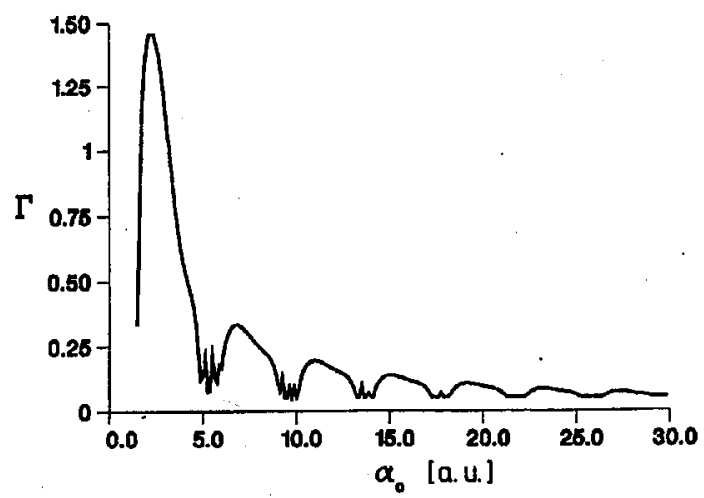

Fig. 4. Ionization rate $\Gamma$ (in inverse optical periods) as a function of oscillation amplitude $\alpha_{0}\left(=A / \omega^{2}\right)$. A limited initial ensemble produces a very narrow distribution, around the phase-space point $u, \dot{u}=(-9,0.39)$, among survivors of the field ramp. The field ramp-up rate is $\mathrm{d} \alpha_{0} / \mathrm{d} t=0.5 /$ optical period $=0.019$ a.u., and $\omega=0.236$ a.u. The ionization distance is taken to be $4 \alpha_{0}$.

Results of our classical calculation are shown in Fig. 4, where the ionization rate $\Gamma$ (in inverse optical periods) is plotted as a function of $\alpha_{0}$. Note that the decrease in $\Gamma$ with increasing $\alpha_{0}$ is not monotonic. The classical results are consistent with the quantum results [14-16], which indicate a minimum lifetime of approximately one optical period and an oscillatory decrease in rate. The precise values of $\Gamma$ and shape of the $\Gamma$ vs. $\alpha_{0}$ curve depend somewhat on the choice of ramp slope and initial ensemble; these determine the ensemble that remains after the end of the ramp. We limit the ensemble that survives the ramp to a distribution localized in phase space; but, qualitatively the results do not depend upon the specific selection of members of the ensemble as long as they are sufficiently clustered in the phase space.

The oscillatory behavior of $\Gamma\left(\alpha_{0}\right)$ can be understood classically as resulting from the combination of the periodic field variation with the near-periodic return of the electron to the vicinity of the nucleus where large momentum transfer can take place to cause ionization [2]. The electron motion will be determined by $\alpha_{0}$ 
and, because only a narrow range of initial conditions in the ensemble survives the ideal adiabatic ramp-up, certain values of $\alpha_{0}$ will make ionization more likely than others due to the resulting coincidence between electron return and high field.

The fact that we find suppression of ionization in a short-range potential does not contradict our earlier results [6], despite our claim therein that there is no stabilization due to interference suppression (in the sense of Fedorov [18-20]) in short-range potentials. That conclusion was based on the fact that short-range potentials have only a finite number of bound states, thus limiting the number of states that can interfere effectively; the result is that the probability of ionization by a pulse goes to unity at high fields in short-range potentials. Careful distinction must be made between probability of ionization and rate of ionization; the apparent absence of stabilization (no reduced ionization probability) in short-range potentials is simply due to a quantitative difference in the integrated ionization rates (see Eq. (1)), compared to those for long-range potentials. There is no qualitative difference between long-range and short-range potentials as far as ionization suppression (reduction of ionization rates) is concerned. By comparing $\Gamma\left(\alpha_{0}\right)$ for a short-range and a Coulomb potential [9], we find that under identical pulsed-field conditions which result in only $\approx 63 \%$ ionization for the long-range potential, there will be $>99 \%$ ionization for the short-range potential. This means that our earlier conclusion of minimal stabilization in a short-range potential is, in fact, consistent with these results showing an ionization rate that decreases with increasing field, because the decrease is significantly slower than that for a long-range potential $[9,14,21]$.

\section{Statistical relative stability of classical orbits}

We study the stability of orbits (solutions to Eqs. (2) and (3)) within the time domain during the turn-on, $0 \leq t_{0} \leq T_{0}$. A difficulty in analyzing stability is that the field, $\varepsilon\left(t_{0}\right)$, is the dominant term in Eqs. (2) and (3) for larger $A$; thus stability at any instant does not necessarily imply stability at later times. A point to note here is that the stability equations (10)-(14), given below, do not involve the field amplitude $A$ explicitly. Therefore, what we find is the instantaneous stability condition of the orbits in the absence of an external field. Here, we take an approach somewhat modified from the conventional one, and define an ionization point (IP) to be that point for an orbit that ionizes within $0 \leq t_{0} \leq T_{0}$ for which the $\rho$-component of the force acting on the particle is maximum. For the overwhelming majority of cases, the IP corresponds to the point of closest approach to the nucleus, after which the particle's $\rho$ coordinate increases monotonically. An example is shown in Fig. 5.

The IP, defined this way with respect to the $\rho$ coordinate, is an expression of the fact that for the ensemble represented here, i.e., for sufficiently large azimuthal angular momentum, the condition for ionization resides almost entirely with respect to the dynamics associated with $\rho$. This can be made plausible by examination of Eqs. (2) and (3). A particle of the ensemble is first accelerated along the $z$ direction in a strong field, then accelerated back toward the nucleus after having reached the $z$ turning point at $|z|=\alpha_{0}$. When the particle reaches the azimuthal plane it is experiencing an acceleration along the $\rho$ direction at the 


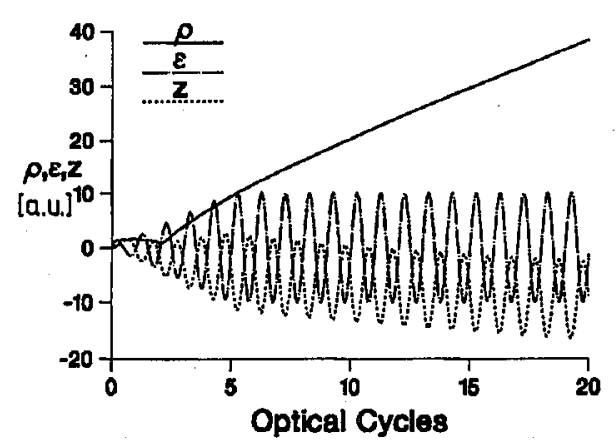

Fig. 5. Field $\varepsilon(t)$ and coordinates $(\rho, z)$ as functions of time for a member of the ensemble that does not survive ramp-up. Here $A=10, m=0.75$, and $R=2$.

same time that the $z$-component of acceleration is small or zero. If the centrifugal term is larger than the Coulomb attraction, the particle will experience an acceleration along the $\rho$ direction, away from the nucleus. This acceleration is larger, the smaller the $\rho$ coordinate, corresponding to the closest approach to the nucleus. We find this to be overwhelmingly the case for the dynamics corresponding to our ensemble. We define a critical point (CP) for an orbit that remains bounded in $0 \leq t_{0} \leq T_{0}$ using the identical criterion, the maximum $\rho$-component of the force acting on the particle. These definitions enable consistent statistical comparison of the relative stability of ionization points and critical points.

Thus we examine the behavior of $\rho=\rho\left(t_{0}\right)+\delta \rho$ and $z=z\left(t_{0}\right)+\delta z$ at an IP or $\mathrm{CP}$, and analyze statistically the relative stability of the points, IP and CP, using the ansatz $\delta \rho \sim \exp \left(\mathrm{i} \omega_{0} t\right), \delta z \sim \exp \left(\mathrm{i} \omega_{0} t\right)$. Equations (2) and (3) become

$$
\begin{aligned}
& \frac{\mathrm{d}^{2} \delta \rho}{\mathrm{d} t^{2}}=-\left.\frac{\partial^{2} V}{\partial \rho \partial z}\right|_{0} \delta z-\left.\frac{\partial^{2} V}{\partial \rho^{2}}\right|_{0} \delta \rho-\left.\frac{3 m^{2}}{\rho^{4}}\right|_{0} \delta \rho, \\
& \frac{\mathrm{d}^{2} \delta z}{\mathrm{~d} t^{2}}=-\left.\frac{\partial^{2} V}{\partial z^{2}}\right|_{0} \delta z-\left.\frac{\partial^{2} V}{\partial \rho \partial z}\right|_{0} \delta \rho,
\end{aligned}
$$

where $\left.\right|_{0}$ indicates evaluation at $\rho_{0}=\rho\left(t_{0}\right)$ and $z_{0}=z\left(t_{0}\right)$. The stability eigenvalue, $\omega_{0}^{2}$, is determined by

$$
\omega_{0}^{4}-B \omega_{0}^{2}+C=0
$$

where

$$
\begin{aligned}
& B=-\frac{\left|V^{\prime}\right|}{r}-\left|V^{\prime \prime}\right|+\frac{3 m^{2}}{\rho^{4}}, \\
& C=\left|V^{\prime}\right| \frac{\left|V^{\prime \prime}\right|}{r}-\frac{3 m^{2}}{\rho^{4}}\left(\left|V^{\prime}\right| \frac{\rho^{2}}{r^{3}}+\left|V^{\prime \prime}\right| \frac{z^{2}}{r^{2}}\right) .
\end{aligned}
$$

An orbit is unstable at the IP if the stability eigenvalue $\omega_{0}^{2}$ is negative or complex. In our study of many potentials with different parameters and initial conditions, all IP have values of $\omega_{0}^{2}<0$ (i.e., $C<0$ when the negative sign of the solution of Eq. (12) is taken). The values of $B$ and $C$ are found by using the potential 
$V(r)=-(1 / r) \exp [-\alpha(r-1)]$. The reason for using this particular functional form is to make a fair comparison between short-range potentials $(\alpha>0)$ and the long-range Coulomb potential $(\alpha=0)$; in both cases we use the same ensemble of 200 sets of initial conditions, and at $r=1$ the kinetic and potential energy will be the same for both types of potential.
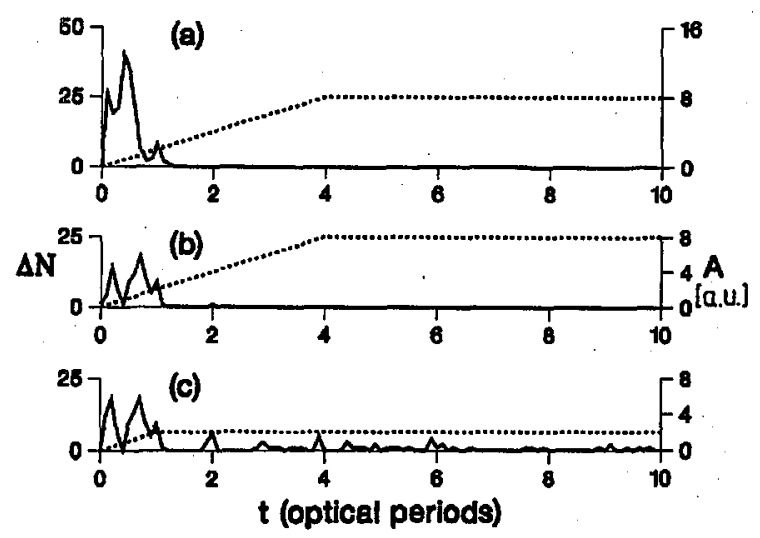

Fig. 6. Number, $\Delta N$, of particles (out of 200) that reach ionization points per one-tenth optical period versus time (solid line); field envelope and plateau value $A$ (dotted line). Graphs (a) and (b) are for short-range $(\alpha=0.2)$ and long-range $(\alpha=0)$ potentials, respectively, with a four-period ramp-up and $A=8$. Graph (c) represents a one-period ramp-up with $A=2$ for the long-range potential.

We consider the probability $P$ of ionization by a pulse with $T-T_{0}=30$ optical periods and $R=2$; we find that a small reduction of the range of the potential can destroy HFIS. For the long-range potential, $P$ decreases with increasing $A$, even though the field amplitude ramps up through the "death valley" (where $A$ has a value around 1 or 2) at the same rate for all $A$, because of the decreasing ionization rate in the plateau region of the pulse. For the short-range potential, $P$ never decreases because there is essentially complete ionization on the ramp-up. This is illustrated in Fig. 6a, where the number of ensemble members reaching an IP in each 0.1-optical-period interval (proportional to the product of the ionization rate and the number remaining unionized) is plotted as a function of time for a fixed value of $A$. For the Coulomb potential, it can be seen from Figs. $6 \mathrm{~b}$ and $\mathrm{c}$ that approximately the same amount of ionization (about $50 \%$ of the particles) occurs on the ramp-up, whether to $A=2$ or to $A=8$. At larger plateau values of $A$, once the particle survives passage through "death valley" we obs: ve that its orbit becomes so large that its motion is controlled by the external field, and it is virtually immune from the atomic force; ionization in the ensemble becomes negligible, as in Fig. $6 \mathrm{~b}$. On the other hand, $A=2$ can produce complete ionization $(P=1)$ because the orbits remain closer to the nucleus, resulting in a larger $\left|\omega_{0}^{2}\right|$ (Eq. (12)); hence consistently unstable orbits arise and continued ionization occurs in the ensemble. Although the pulses are completed, and ionization 

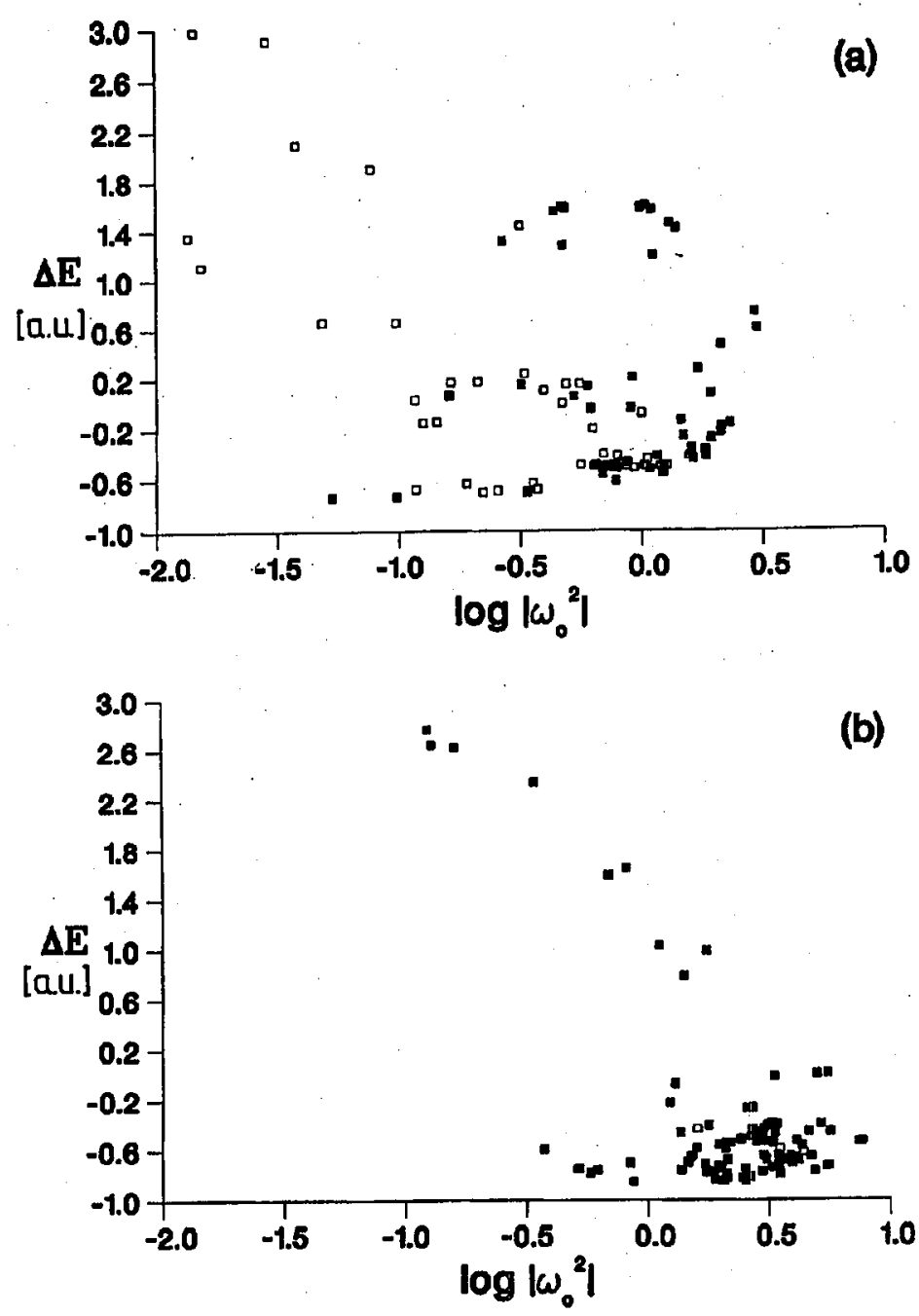

Fig. 7. Energy input $\Delta E$, as measured at the ionization point or critical point, versus $\log _{10}\left(\left|\omega_{0}^{2}\right|\right)$ for (a) long-range $(\alpha=0)$ and (b) short-range $(\alpha=0.3)$ potentials. Solid squares $(\square)$ represent ionized particles and hollow squares $(\square)$ are survivors.

determined afterward, Fig. 6 does not show the ramp-down; as we pointed out earlier [6], ionization during pulse turn-off is negligible. We now understand this to be a result of the orbits not having time to return close to the nucleus before the field passes through the region around $A \approx 1-2$. It is evident statistically that the size of $\left|\omega_{0}^{2}\right|$ can indicate a measure of instability and likelihood of ionization, despite the obvious limitations of the method. The application of the method in the case of a short-range potential demonstrates its utility; for $A=8$, there is complete ionization, in contrast to the Coulomb case. We can see from Fig. 6 that 
the reason for this is that the ionization rate, for a given value of field amplitude on the ramp-up, is significantly larger for the short-range potential. The analysis of stability explains this, as is illustrated in Fig. 7. Each point in Figs. 7a and b reflects the state at an IP or CP. The overwhelming majority of orbits that ionize have larger $\left|\omega_{0}^{2}\right|$ at that point than orbits that do not ionize. Another minor indicator of stability is $\Delta E$, the energy input, i.e., the difference between the particle's energy at the IP or CP and its energy at $t=0$. A very large $\Delta E$ pumps the orbit to ionization even when the particle is located far away from the nucleus and $\left|\omega_{0}^{2}\right|$ is small. A comparison of parts (a) and (b) of Fig. 7 shows that as $\alpha$ increases and the range of the potential becomes shorter, both $\left|\omega_{0}^{2}\right|$ and $\Delta E$ are pushed toward larger values, enhancing the ionization.

Equations (12) and (14) explain why $\left|\omega_{0}^{2}\right|$ can serve the purpose of predicting ionization. For $C$ to be negative at an IP, the value of the coordinate $\rho_{0}$ should be small or $z_{0}$ should be large. A small $\rho_{0}$ implies a large azimuthal angular speed $\dot{\theta}_{0}$ from $\dot{\theta}_{0}=m / \rho_{0}^{2}$. A larger $\dot{\theta}_{0}$ coupled with a short-range force of diminishing influence over the orbits moving away from the nucleus, causes ionization, as expected. This same physical picture is also applicable to explain the presence of HFIS for the Coulomb potential. In this case, when the plateau amplitude of the field pulse is at $A=2$ (near the "death valley" region of high ionization rate), those particles not ionized in their first pass at small $\rho_{0}$ can try again, whereas at $A=8$, the orbits escape safely to a larger $\rho$ (smaller $\left.\left|\omega_{0}^{2}\right|\right)$, where they stay as relatively more stable orbits. Our extensive numerical investigation, however, demonstrates that all orbits are unstable throughout most of their dynamical history. This is an important point. We have examined many pairs of orbits, originating from two very close initial conditions resulting in only slightly different $\dot{\theta}_{0}$ or $\left|\omega_{0}^{2}\right|$, in which one ionizes and one remains bound after reaching the IP or CP. This explains why ionized and unionized orbits cannot be clearly distinguished in a simple way based on the initial conditions, much as the initial conditions determine regular or chaotic behavior in the model of Ref. [22].

Our explanation of the importance of $\left|\omega_{0}^{2}\right|$ in producing ionization, as shown in Figs. $7 \mathrm{a}$ and $\mathrm{b}$, is a statistical statement. There are obviously a number of exceptions because of other factors that contribute to the probability of ionization at an IP or CP: the position coordinate $z_{0}$, which determines $\Delta E$; the time, which determines the phase of the field at the IP or CP; and the direction of the particle's velocity there. These minor factors are difficult to correlate to form a cohesive deterministic picture. We account for them through their distribution over the ensemble, giving us a statistical interpretation.

\section{Conclusions}

The role of the ramp-up of the external field in HFIS was analyzed. The realization of an optimum turn-on rate, $R$, provides an essential clue to the explanation of HFIS using the classical ensemble. The results have led to the use of a weak prepulse to enhance HFIS, and suggest the expediency to implement an optimization procedure within suitable constraints.

We point out the need to distinguish between two different interpretations of HFIS. One interpretation, applicable to the case of pulsed fields, is that HFIS 
is a reduction in the probability of ionization with increasing pulse intensity. The other, for quasi-steady fields, is that HFIS is a reduction in the ionization rate with increasing field amplitude. The major features in either case are that high field amplitudes induce less ionization, and that ionization is more likely for a particle bound in a short-range potential. We show that these features may be explained in terms of a classical dynamical ensemble. We show that orbits in general are characterized by a stability eigenvalue $\left(\omega_{0}^{2}\right)$ that is a good indicator of whether ionization will occur. Our results show that there exist points (IP and CP) in configuration space at which the particle experiences an impulse; a short-range potential is more likely than a long-range potential to lose its grip on the particle at the IP or CP, meaning that ionization is more likely in the short-range case. This means that at any value of field amplitude, the ionization rate will be larger for a short-range potential than for a long-range potential; this quantitative difference in instantaneous rates; acting over the duration of a pulse, can result in a qualitative difference in the resultant ionization probabilities.

The question as to just why the classical model gives results equivalent to those of the quantum approach, even for a short-range potential, merits further investigation. In earlier work [6], we suggested that the classical-quantum equivalence for a long-range potential derived from the importance of interference and coherent superpositions of many quantum bound states out of the infinite number available $[18-20]$. For the short-range potential, our preliminary suspicion is that interference is again responsible, although it is less effective because the number of the bound states of the potential, and therefore the number of $\mathrm{K}-\mathrm{H}$ eigenstates for finite $\alpha_{0}$, is finite. Evidence pointing to interference can be found in Ref. [14], where the initial decline in the ground-state ionization rate begins shortly after the appearance of the first light-induced excited state. Further evidence for the role of interference between $\mathrm{K}-\mathrm{H}$ eigenstates may be found in the existence (absence) of ionization suppression for a one-dimensional (three-dimensional) delta-function potential $[2,23-25]$. An explanation for this behavior of delta-function potentials may be found by applying the method used in Ref. [6], for estimating the number of bound states, to the corresponding time-averaged $(\mathrm{K}-\mathrm{H})$ potentials. It is found that the number of bound states of the $\mathrm{K}-\mathrm{H}$ potential derived from a one- (three-) dimensional delta function goes to infinity (zero) as $\alpha_{0}$ increases without limit in the three-dimensional case, there are no light-induced excited states to interfere. For the Gaussian potential treated here, agreement with the quantum results also suggests that the field-induced superposition of $\mathrm{K}-\mathrm{H}$ eigenstates is not dominated by nonclassical interferences, in which the Wigner quasi-probability distribution takes on negative values [26]. Thus we suggest that these quantum-classical correspondences point to interference as the mechanism underlying HFIS even in the adiabatic limit.

\section{References}

[1] J.H. Eberly, K.C. Kulander, Science 262, 1229 (1993).

[2] N.B. Delone, V.P. Krainov, Usp. Fiz. Nauk 165, 1295 (1995) [Phys. Usp. 38, 1247 (1995)].

[3] B. Ritchie, C.M. Bowden, C.C. Sung, Y.Q. Li, Phys. Rev. A 41, 6114 (1990). 
[4] C.M. Bowden, C.C. Sung, S.D. Pethel, A.B. Ritchie, Phys. Rev. A 46, 592 (1992).

[5] C.M. Bowden, S.D. Pethel, C.C. Sung, J.C. Englund, Phys. Rev. A 46, 597 (1992).

[6] M. Dombrowski, A.T. Rosenberger, C.C. Sung, Phys. Lett. A 199, 204 (1995).

[7] C.C. Sung, A.T. Rosenberger, S.D. Pethel, C.M. Bowden, in: Super-Intense Laser-Atom Physics IV, Eds. H.G. Muller, M.V. Fedorov, Kluwer Academic Publishers, Dordrecht 1996, p. 73.

[8] S.D. Pethel, C.M. Bowden, C. C. Sung, Laser Phys. 7, 558 (1997).

[9] A.T. Rosenberger, C.C. Sung, S.D. Pethel, C.M. Bowden, Laser Phys. 7, 563 (1997).

[10] A.T. Rosenberger, C.C. Sung, S.D. Pethel, C.M. Bowden, Phys. Rev. A, to be published.

[11] T. Grochmalicki, M. Lewenstein, K. Rzazewski, Phys. Rev. Lett. 66, 1038 (1991).

[12] K.C. Kulander, K.J. Schafer, J.L. Krause, Phys. Rev. Lett. 66, 1038 (1991).

[13] F. Benvenuto, G. Casati, D.L. Shepelyansky, Phys. Rev. A 45, R7670 (1992).

[14] M. Marinescu, M. Gavrila, Phys. Rev. A 53, 2513 (1996).

[15] G. Yao, S.-I. Chu, Phys. Rev. A 45, 6735 (1992).

[16] A. Fearnside, R.M. Potvliege, R. Shakeshaft, Phys. Rev. A 51, 1471 (1995).

[17] T. Millack, J. Phys. B 26, 4777 (1993).

[18] M.V. Fedorov, A.M. Movsesian, J. Opt. Soc. Am. B 6, 928 (1989).

[19] M.V. Fedorov, A.M. Movsesian, J. Opt. Soc. Am. B 6, 1504 (1989).

[20] M.V. Fedorov, Comments At. Mol. Phys. 27, 203 (1992).

[21] M. Pont, M. Gavrila, Phys. Rev. Lett. 65, 2362 (1990).

[22] F. Benvenuto, G. Casati, D.L. Shepelyansky, Z. Phys. B 9, 481 (1994).

[23] S. Geltman, Phys. Rev. A 45, 5293 (1992).

[24] V.P. Krainov, M.A. Preobrazhenskii, Zh. Eksp. Teor. Fiz. 103, 1143 (1993) [Sov. Phys. JETP 76, 559 (1993)].

[25] H.R. Reiss, J. Opt. Soc. Am. B 13, 355 (1996).

[26] J.B. Watson, C.H. Keitel, P.L. Knight, K. Burnett, Phys. Rev. A 52, 4023 (1995). 